\title{
Suppression effect of thyme and carvacrol nano-emulsions on Aspergillus fumigatus isolated from patients in the intensive care unit of Assiut University Hospital, Egypt
}

\author{
Alshimaa A. Hassanien ${ }^{1}$, Walaa M. Elsherif2 ${ }^{(D)}$, Rasha Hamed ${ }^{3}$ (D) and Asmaa A. A. Hussein ${ }^{4}$
}

1. Department of Zoonoses, Faculty of Veterinary Medicine, Sohag University, Sohag, Egypt; 2. Nanotechnology Research Unit, Animal Health Research Institute, Agriculture Research Centre, Egypt; 3. Department of Anaesthesia, Intensive

Care Unit and Pain Management, Faculty of Medicine, Assiut University, Egypt; 4. Molecular Biology Research and Studies Institute, Assiut University, Egypt.

Corresponding author: Alshimaa A. Hassanien, e-mail: Hassanien2008@yahoo.com

Co-authors: WME: me.elsherif@yahoo.com, RH: rashaahmed11@aun.edu.eg, AAAH: asmaah@aun.edu.eg

Received: 28-01-2021, Accepted: 23-03-2021, Published online: 19-05-2021

doi: www.doi.org/10.14202/IJOH.2021.116-121 How to cite this article: Hassanien AA, Elsherif WM, Hamed R, Hussein AAA (2021) Suppression effect of thyme and carvacrol nano-emulsions on Aspergillus fumigatus isolated from patients in the intensive care unit of Assiut University Hospital, Egypt, Int. J. One Health, 7(1): 116-121.

\begin{abstract}
Background and Aim: Aspergillus fumigatus is a zoonotic fungus that causes several diseases in humans ranging from allergic reaction to fatal disseminated invasive infection, especially in immunocompromised patients. This study aimed to investigate the incidence of invasive A. fumigatus in patients admitted to the intensive care unit (ICU) of Assiut University Hospital, highlight the factors associated with their infection, and determine the antifungal effect of thyme nano-emulsion (TNE) and carvacrol nano-emulsion (CNE) on isolated A. fumigatus strains.

Materials and Methods: Mycological culture method and scanning electron microscopy (SEM) were used in the identification of A. fumigatus in 630 blood samples collected from 210 patients. TNE and CNE at five concentrations (1\%, $2 \%, 4 \%, 6 \%$, and $8 \%$ ) and average sizes of 90.3 and $75.6 \mathrm{~nm}$, respectively, were characterized by transmission electron microscopy. Their effect on A. fumigatus isolate growth was evaluated by the well-diffusion method and SEM, which was used for the detection of the degenerative effect of $A$. fumigatus ultrastructure.

Results: A. fumigatus was detected in 54 of 210 (25.7\%) patients in the ICU. Advanced age and chronic diseases were considered important risk factors for invasive aspergillosis, especially in patients with more than 1 clinical disease. TNE and CNE showed an inhibitory effect on A. fumigatus isolates, which significantly increased with high concentrations. The respective values for TNE at concentrations of $6 \%$ and $8 \%$ were $6 \pm 0.41 \mathrm{~mm}$ and $15 \pm 0.67 \mathrm{~mm}$. CNE completely inhibited A. fumigatus growth at concentrations of $4 \%, 6 \%$, and $8 \%$, while mean inhibition zones of $22 \pm 0.68 \mathrm{~mm}$ and $30 \pm 0.32 \mathrm{~mm}$ appeared at concentrations of $1 \%$ and $2 \%$. SEM demonstrated degenerative changes in A. fumigatus structure.
\end{abstract}

Conclusion: TNE and CNE can be used in bioactive treatments against $A$. fumigatus, and additional studies are required to determine the safe and effective doses and best method for application in human and veterinary medicine.

Keywords: Aspergillus fumigatus, carvacrol nano-emulsion, intensive care unit patients, invasive aspergillosis, scanning electron microscopy, thyme nano-emulsion.

\section{Introduction}

Aspergillus species are widely spread in soil in which they inhabit as saprophytes. They have the ability to infect several living hosts, such as insects, animals, birds, plants, and human, causing fatal disseminated diseases in both animals and human [1]. A wide range of clinical conditions are caused by aspergillosis infection, ranging from allergic reactions to disseminated invasive aspergillosis (IA), especially in immunocompromised patients. Recently, Aspergillus fumigatus is considered the most common cause of IA. It is ubiquitous in the environment

Copyright: Hassanien, et al. This article is an open access article distributed under the terms of the Creative Commons Attribution 4.0 International License (http://creativecommons.org/licenses/ by/4.0/), which permits unrestricted use, distribution, and reproduction in any medium, provided you give appropriate credit to the original author(s) and the source, provide a link to the Creative Commons license, and indicate if changes were made. The Creative Commons Public Domain Dedication waiver (http:// creativecommons.org/ publicdomain/zero/1.0/) applies to the data made available in this article, unless otherwise stated. with small spores, which enhance its penetration and colonization to airways, especially in patients with compromised lung mucosal defense. This allows its spread into other organs, causing hematogenous fungal diseases [2-4].

IA is an emerging disease in patients in the intensive care unit (ICU), even without displaying apparent immunodeficiency, with a mortality rate of $>80 \%$. Factors that enhance the development of IA are chronic obstructive pulmonary disease (COPD), longterm corticosteroid treatment, HIV infection, lung transplant, liver cirrhosis, and malnutrition [5].

Antifungal drugs have a low effect in IA, as they are degraded in the bloodstream and do not directly reach the infected organ. Therefore, using nanoparticles as a drug delivery system may increase its viability [6]. Recently, antifungal-resistant isolates developed; thus, several studies are important to find accurate drugs and discover new effective materials for the treatment of fungal diseases [7]. 
Essential oils are naturally volatile and non-volatile compounds produced by aromatic plants. Thyme oil resulted from extraction of Thymus vulgaris in which thymol is the main component. Carvacrol compound was found in some aromatic plants, such as oregano, wild bergamot, and pepperwort. It is displayed in several pharmaceutical and biological fields as antioxidant, antifungal, anti-inflammatory, antibacterial, and vasorelaxant agents. Therefore, they are recently applied in different fields, such as pharmaceutical industries, cosmetics, and food [8,9]. Essential oil utilization is limited as they are low water-soluble. This problem can be resolved by encapsulation of essential oils in oil/water emulsions or water/oil nano-emulsions [10].

Nano-emulsions with nanosize are stable against coalescence, creaming, and flocculation, which enable them to be used in food industry and delivery systems for drugs, vitamins, and flavors [11]. It enhances the bioavailability of bioactive and hydrophobic drugs, in which they are characterized by improper absorption and slow oral bioavailability [12]. Thus, several studies in the application of nano-emulsions in agriculture and nanomedicine are important to determine the most effective and safe treatment methods for human.

Therefore, this study aimed to investigate the incidence of A. fumigatus in patients in the ICU and use two natural compounds, namely, thyme nano-emulsion (TNE) and carvacrol nano-emulsion (CNE), as therapeutic agents against isolated strains of $A$. fumigatus.

\section{Materials and Methods}

\section{Ethical approval and Informed consent}

Ethical approval was obtained from our local ethical committee of Assiut University Hospital. Patients' participation was optional and collection of samples and data was done after their consent or their guardian consent.

\section{Study period and location}

This study was conducted from December 2019 to January 2021. All samples and data were collected from patients admitted to Assiut University Hospital. The samples were transferred in an insulated icebox to the Faculty of Veterinary Medicine, Sohag University, for the laboratory work.

\section{Data and sample collection}

As A. fumigatus existence in the blood is intermittent, 630 blood samples were collected from 210 patients in the ICU (three samples from each patient represented one sample) with chronic diseases, such as respiratory disease, renal failure, liver cirrhosis, infected vascular diseases, diabetes mellitus (DM), hepatitis C, and cardiac disease. Data were collected from patients by standard form, including age, sex, and medical history.

\section{Mycological examination}

Potato dextrose agar (PDA) with antibiotics was cultured by patient's blood samples for 5 days at $25^{\circ} \mathrm{C}$. A. fumigatus was identified macroscopically and microscopically and then by scanning electron microscopy (SEM) (JEOL, JSM-5400LV, Japan) in Electron Microscope Unit of Assiut University [13].

\section{TNE and CNE preparation}

At room temperature $\left(25^{\circ} \mathrm{C}\right)$, Tween $802 \mathrm{v} / \mathrm{v} \%$ was dissolved in double-distilled water. Then, a magnetic stirrer (Daihan, Korea) was used in shaking the mixture for $10 \mathrm{~min}$ to obtain a homogenous solution. The essential oils were slowly added and mixed for 15 min using direct-driven stirrer. Subsequently, the resulting emulsions were sonicated with ultrasonic homogenizer $(25 \mathrm{kHz}, 650 \mathrm{~W})$ [9]. The nano-emulsions were prepared in Nanotechnology Research Unit, Animal Health Research Institute, Assiut, Egypt. The size of both TNE and CNE was measured in the Electron Microscopy Unit, Assiut University, using TEM (Jeol, USA).

\section{TNE and CNE effect}

A. fumigatus isolates $(\mathrm{n}=54)$ were cultured for 6 days at $25^{\circ} \mathrm{C}$ in liquid potato dextrose medium; then, $1 \times 10^{6} \mathrm{CFU} / \mathrm{mL}$ was inoculated on PDA containing $50 \mu \mathrm{L}$ of the prepared nano-emulsions with different concentrations $(1 \%, 2 \%, 4 \%, 6 \%$, and $8 \%$ ) on each well and incubated at $25^{\circ} \mathrm{C}$ for 6 days. Nano-emulsion free plates of PDA were applied as control (Figure-1). The antifungal effect of nano-emulsions was detected by inhibition zone growth [14]. Then, SEM was used for the identification of nano-emulsions effect on the fungal ultrastructure.

\section{Statistical analysis}

SPSS 14 (IBM Corp., NY, USA) was used for describing the antimicrobial effect of TNE and CNE on A. fumigatus isolates by mean and standard error. A significant difference was indicated by $\mathrm{p}<0.05$.

\section{Results}

\section{A. fumigatus detection and risk factors}

Of 210 patients in the ICU, $54(25.7 \%)$ were positive for A. fumigatus. Patients aged $>60$ years

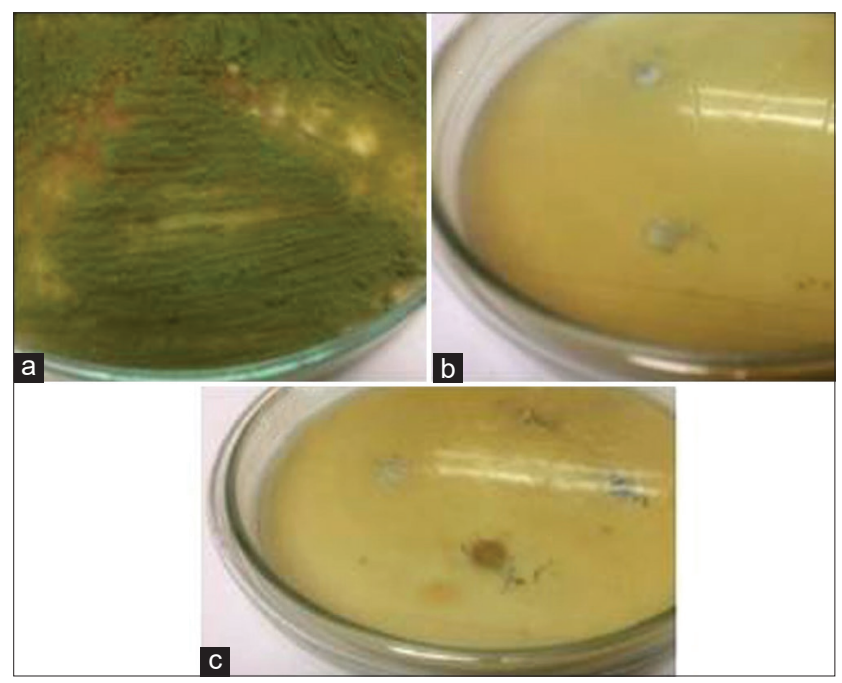

Figure-1: Aspergillus fumigatus in potato dextrose agar plates: (a) Positive control, (b) negative control for thyme nanoemulsion, (c) negative control for carvacrol nano-emulsion. 
represented the highest infection rate $(34.6 \%)$, followed by those aged 51-60 (23.8\%), 41-50 (17.8\%), and $30-40(14.3 \%)$ years. The infection rates were $26.8 \%$ in men and $24.1 \%$ in women (Table-1). Based on the medical history of patients, the highest percentage of A. fumigatus was noted in patients with COPD, liver cirrhosis, and hepatitis C (42.1\%), followed by patients with COPD, cardiac diseases, and DM (38.9\%); respiratory and rheumatic heart diseases $(37.9 \%)$; cardiac and vascular surgeries $(35 \%)$; cardiac diseases and renal failure (29.4\%); renal failure (19.2\%); infected vascular surgeries (17.6\%); and liver cirrhosis $(11.8 \%)$. The lowest result $(10 \%)$ was reported in patients with cardiac diseases (Table-2).

\section{TNE and CNE effect}

TNE has an inhibitory effect on $A$. fumigatus at concentrations of $6 \%$ and $8 \%$, with mean inhibition zones of $6 \pm 0.41 \mathrm{~mm}$ and $15 \pm 0.67 \mathrm{~mm}$, respectively. Complete resistance was detected at concentrations of $1 \%, 2 \%$, and $4 \%$. Moreover, CNE represented inhibitory effect on fungal growth at concentrations of $1 \%$ and $2 \%$ with mean inhibition zone of $22 \pm 0.68 \mathrm{~mm}$ and

Table-1: Incidence of Aspergillus fumigatus among intensive care unit patients.

\begin{tabular}{lccccc}
\hline $\begin{array}{l}\text { Patients } \\
\text { characteristics }\end{array}$ & $\begin{array}{c}\text { Blood } \\
\text { samples } \\
\mathbf{n = 2 1 0}\end{array}$ & & \multicolumn{2}{c}{$\begin{array}{c}\text { Patients with } \\
\text { Aspergillus } \\
\text { fumigatus } \\
\mathbf{n = 5 4}\end{array}$} \\
\cline { 2 - 3 } \cline { 5 - 6 } & $\mathbf{n}$ & \% & & $\mathbf{n}$ & \% \\
\hline Age & 21 & 10 & & 3 & 14.3 \\
$30-40$ & 45 & 21.4 & & 8 & 17.8 \\
$41-50$ & 63 & 30 & & 15 & 23.8 \\
$51-60$ & 81 & 38.6 & & 28 & 34.6 \\
>60 & & & & & \\
Gender & 127 & 60.5 & & 34 & 26.8 \\
Male & 83 & 39.5 & & 20 & 24.1 \\
Female & & & & &
\end{tabular}

Table-2: Medical history of Aspergillus fumigatus patients.

\begin{tabular}{|c|c|c|c|c|}
\hline \multirow[t]{2}{*}{ Medical history } & \multicolumn{2}{|c|}{$\begin{array}{c}\text { Blood } \\
\text { samples } \\
n=210\end{array}$} & \multicolumn{2}{|c|}{$\begin{array}{c}\text { Patients with } \\
\text { Aspergillus } \\
\text { fumigatus } \\
\mathrm{n}=54\end{array}$} \\
\hline & $\mathbf{n}$ & $\%$ & $\mathbf{n}$ & $\%$ \\
\hline Infected vascular surgeries & 34 & 16.2 & 6 & 17.6 \\
\hline Cardiac diseases & 30 & 14.3 & 3 & 10 \\
\hline Renal failure & 26 & 12.4 & 5 & 19.2 \\
\hline Liver cirrhosis & 17 & 8.1 & 2 & 11.8 \\
\hline $\begin{array}{l}\text { Respiratory diseases and } \\
\text { rheumatic heart disease* }\end{array}$ & 29 & 13.8 & 11 & 37.9 \\
\hline $\begin{array}{l}\text { COPD, liver cirrhosis, and } \\
\text { hepatitis C* }\end{array}$ & 19 & 9.05 & 8 & 42.1 \\
\hline $\begin{array}{l}\text { Cardiac and vascular } \\
\text { surgeries* }\end{array}$ & 20 & 9.5 & 7 & 35 \\
\hline $\begin{array}{l}\text { Cardiac diseases and renal } \\
\text { failure* }\end{array}$ & 17 & 8.1 & 5 & 29.4 \\
\hline $\begin{array}{l}\text { COPD, cardiac diseases, } \\
\text { and DM* }\end{array}$ & 18 & 8.6 & 7 & 38.9 \\
\hline
\end{tabular}

*Patients have more than 1 disease, COPD $=$ Chronic obstructive pulmonary disease, $\mathrm{DM}=$ Diabetes Mellitus
$30 \pm 0.32 \mathrm{~mm}$, respectively. Complete suppression of A. fumigatus growth was reported in concentrations of 4,6 , and $8 \%$ (Table-3). SEM described the damage of A. fumigatus ultrastructure due to the effect of TNE and CNE (Figure-2).

\section{Discussion}

Table-1 reveals that $A$. fumigatus was detected in the blood of patients in the ICU with percentage of $25.7 \%$. Higher results were reported by Peláez et al. [15] and Steinmann et al. [16]. The lower results of blood culture may be attributed to that the blood culture only detected the existence fungal hyphae in blood circulation and, in case of IA, only the detached fungal hyphae that enter the bloodstream can be detected, so its presence in the circulation is intermittent [17].

A. fumigatus infection was increased in patients with old age and decreased in patients with younger age. It was detected with highest percentage in patients aged $>60$ years. Therefore, advanced age is considered an important factor in IA [18]. Patients with more than 1 disease represented the highest infection rate, especially those with respiratory diseases (Table-2). From our results, patients with COPD, liver cirrhosis, and hepatitis $\mathrm{C}$ represented the highest percentage of A. fumigatus (42.1\%). According to Chen et al. [19], the incidence of IA was increased in decompensated liver cirrhosis, neutropenia, pulmonary diseases, and diabetes. The majority of patients received antibiotics and steroids in hospitals to prevent inflammation and infections, but they decrease human immune function and do not cover all pathogens; thus, patients are prone to several pathogens, such as Aspergillus species. Evidence of aspergillosis was increased with some risk factors, such as chronic lung diseases, liver failure, surgery, immaturity, and sepsis. Inhalation of A. fumigatus resulted in several diseases, depending on human immunological status, which varies from slight hypersensitivity to serious invasive infection in internal organs [1].

Normal air contains a high concentration of Aspergillus due to their high capacity of sporulation, resulting in conidia of small size $(2-3 \mu \mathrm{m})$, which enables them to reach the lung parenchyma through

Table-3: Suppression effect of thyme and carvacrol nano-emulsions on Aspergillus fumigatus isolates.

\begin{tabular}{|c|c|c|c|}
\hline \multirow[t]{3}{*}{ Concentration } & \multicolumn{3}{|c|}{ Inhibition zone $(\mathrm{mm})$} \\
\hline & $\begin{array}{c}\text { Thyme } \\
\text { nano- } \\
\text { emulsion }\end{array}$ & $\begin{array}{c}\text { Carvacrol } \\
\text { nano-emulsion }\end{array}$ & p-value \\
\hline & Mean \pm SdE & Mean \pm SdE & \\
\hline $1 \%$ & $\mathrm{NZ}$ & $22 \pm 0.68$ & 0.05 \\
\hline $2 \%$ & $N Z$ & $30 \pm 0.32$ & \\
\hline $4 \%$ & $N Z$ & No growth & \\
\hline $6 \%$ & $6 \pm 0.41$ & No growth & \\
\hline $8 \%$ & $15 \pm 0.67$ & No growth & \\
\hline
\end{tabular}

Mean \pm SdE $=$ Mean \pm standard error, $* \mathrm{NZ}=$ No zone formed 

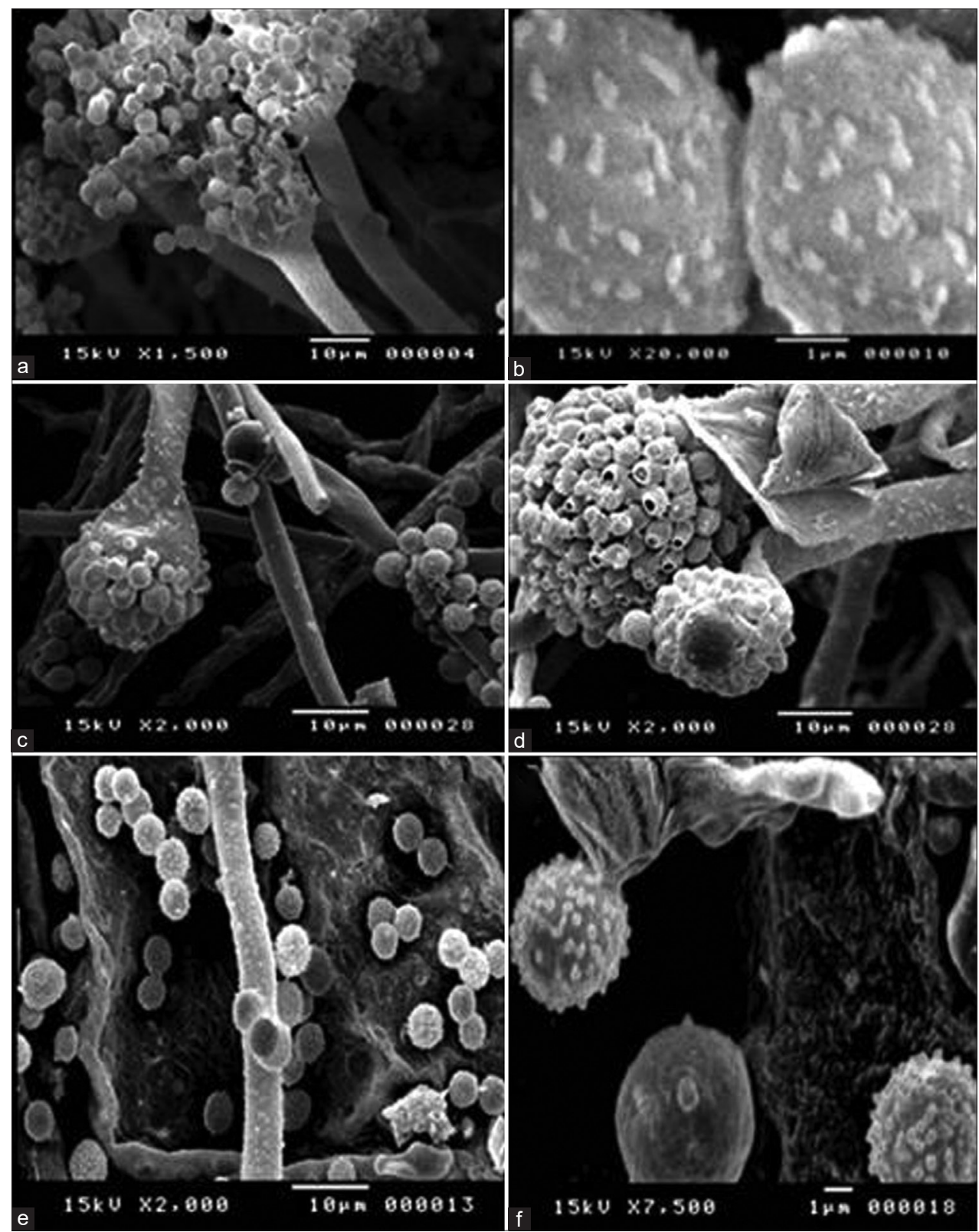

Figure-2: Scanning electron microscopy for ( $A$ and B) untreated Aspergillus fumigatus, (C and D) effect of thyme nanoemulsion at $6 \%$ on $A$. fumigatus, and ( $E$ and $F$ ) effect of CNE at $1 \%$ on $A$. fumigatus.

the airways [20]. Host defense against inhaled fungal spores acts through two lines: The first starts in the mucous layer and ciliary action of the respiratory tract, and the second comprises the phagocytic system of alveolar macrophage. Normally, macrophages, on identifying the beta-D-glucan of the fungal cell wall, produce inflammatory mediators, which stimulate neutrophils to start cellular immunity. In case of chronic diseases, immunosuppression resulted in neutrophil dysfunction and decreased its level. Moreover, Aspergillus secretes toxic metabolites, which interfere with macrophage action and neutrophil phagocytosis, such as mycotoxins, ochratoxins, and aflatoxins [21]. Therefore, disruption of host defense mechanism facilitates the attachment of the fungus into the lung parenchyma and dissemination into other organs with prolonged immunity impairment.

\section{Nano-emulsions effect}

Nano-emulsions have gained attention as antimicrobial agents, so they are applied in the delivery of drugs through the parenteral, oral, transdermal, and transmucosal routes and effectively enhance their bioavailability [22]. They are also used for delivering bioactive food ingredients, which facilitate their solubilization and physiochemical stability in gastrointestinal tract [12].

TNE with an average size of $90.3 \mathrm{~nm}$ and CNE with an average size of $75.6 \mathrm{~nm}$ (Figure-3) were used in five concentrations, and their impact is described in Table3. CNE at $1 \%$ and $2 \%$ inhibited A. fumigatus growth, while complete suppression of growth was represented in concentrations of $4 \%, 6 \%$, and $8 \%$. However, TNE's inhibitory effect was observed only at concentrations of $6 \%$ and $8 \%$. The effect of both nano-emulsions significantly increased with concentration $(\mathrm{p}<0.05)$. 


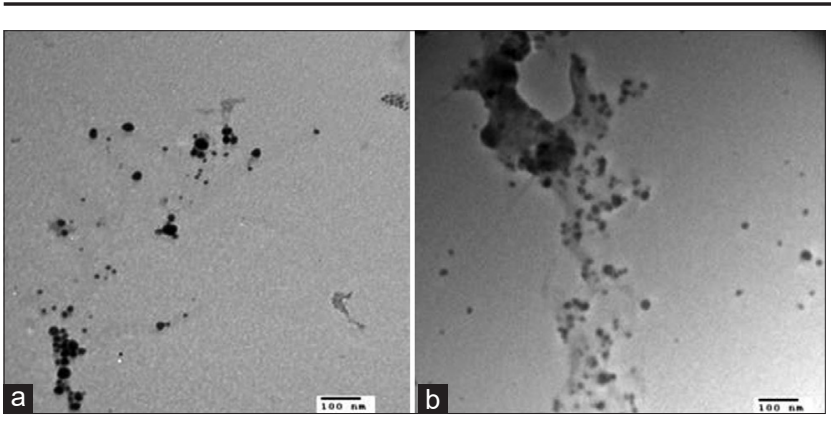

Figure-3: Transmission electron microscopy showing (A) thyme nano-emulsion with average size $90.3 \mathrm{~nm}$, (B) carvacrol nano-emulsion with average size $75.6 \mathrm{~nm}$.

The improvement of CNE formulation in drug delivery makes it more potent for the treatment of several diseases, because nanoformulation in liposomes can solve many problems of confrontation, such as solubility, bioavailability, and stability [23]. Moreover, CNE increases the capacity level of total antioxidant and prevents lipid peroxidation inside the cell [24].

The antimicrobial effect of volatile compounds may be attributed to their lipophilicity, as they can perforate the cell membrane, disrupt cell metabolism, and increase membrane fluidity and permeability. Moreover, they prohibit respiration, disrupt membrane protein function, induce ion leakage, and alter the ion transportation process in the fungal cell [25].

SEM was used to assess the effect of TNE and $\mathrm{CNE}$ on A. fumigatus ultrastructure by describing their morphology surface (Figure-2). The untreated fungi showed abundant growth and strong hyphal attachment with conidia. CNE at lower concentration (1\%) resulted in changes in conidia structure with irregular, loose, and rough structure and distortion in its surface, damage of the fungal hyphae, and fallout of conidia. Treatment with TNE represented limited development and distortion of fungal hyphae. These results are in agreement with Nguefack et al. [26], who found complete inhibition of conidial germination and mycelial growth of $A$. fumigatus using different essential oils. Ergosterol is the main sterol present in the cytoplasmic membrane of $A$. fumigatus. The modifications in its morphology, which resulted from the effect of TNE and CNE, might be linked to the interference in cell wall synthesis, which affects fungal growth and morphology [27]. Therefore, nano-emulsions can be effective antifungal agents. They resulted in morphological deformation, collapse, and deterioration of fungal conidia and hyphae [28].

\section{Conclusion}

The presence of $A$. fumigatus in the blood of patients in the ICU may comprise invasive infection, especially in patients with advanced age and those with more than 1 disease. TNE and CNE as natural compounds represent the antifungal effect on A. fumigatus growth, but CNE has a suppression effect in low concentrations. Therefore, they can be used in various fields associated with medicine, such as antifungal and bioactive supplements.

\section{Authors' Contributions}

AAH: Conceived the idea and designed the study. RH: Collected data and samples. AAH: Analyzed the data and performed the laboratory work. WME: Prepared and characterized the nano-emulsions. AAH and RH: Drafted the manuscript. AAAH Supervised and revised the manuscript. All authors have approved the final manuscript.

\section{Acknowledgments}

The authors thank the Zoonoses Department, Faculty of Veterinary Medicine, Sohag University, for providing their laboratory facilities to perform this work. The authors did not receive any funds for this study.

\section{Competing Interests}

The authors declare that they have no competing interests.

\section{Publisher's Note}

Veterinary World (Publisher of International Journal of One Health) remains neutral with regard to jurisdictional claims in published institutional affiliation.

\section{References}

1. Seyedmousavi, S., Guillot, J., Arné, P., De Hoog, G., Mouton, J., Melchers, W. and Verweij, P. (2015) Aspergillus and aspergilloses in wild and domestic animals: A global health concern with parallels to human disease. Med. Mycol., 53(8): 765-797.

2. Rosa, C., Araujo, R., Rodrigues, A., Pinto-de-Sousa, M. and Pina-Vaz, C. (2011) Detection of Aspergillus species in BACTEC blood cultures. J. Med. Microbiol., 60(Pt 10): 1467-1471.

3. Barton, R.C. (2013) Laboratory diagnosis of invasive aspergillosis: From diagnosis to prediction of outcome. Scientifica (Cairo), 2013: 459405.

4. Romero, M., Messina, F., Marin, E., Arechavala, A., Depardo, R., Walker, L., Negroni, R. and Santiso, G. (2019) Antifungal resistance in clinical isolates of Aspergillus spp.: When local epidemiology breaks the norm. J. Fungi (Basel), 5(2): 41

5. Bassetti, M., Righi, E., De Pascale, G., De Gaudio, R., Giarratano, A., Mazzei, T., Morace, G., Petrosillo, N., Stefani, S. and Antonelli, M. (2014) How to manage aspergillosis in non-neutropenic intensive care unit patients. Crit. Care, 18(4): 458.

6. Barnard, A.S. (2016) Challenges in modelling nanoparticles for drug delivery. J. Phys. Condens. Matter, 28(2): 023002.

7. YienIng, L., Zin, M., Sarwar, A. and Katas, H. (2012) Antifungal activity of chitosan nanoparticles and correlation with their physical properties. Int. J. Biomater., 2012: 632698.

8. Suntres, Z., Coccimiglio, J. and Alipour, M. (2015) The bioactivity and toxicological actions of carvacrol. Crit. Rev. Food Sci. Nutr., 55(3): 304-318.

9. Moradi, S. and Barati, A. (2019) Essential oils nano-emulsions: Preparation, characterization and study of antibacterial activity against Escherichia coli. Int. J. Nanosci. Nanotechnol., 15(3): 199-210.

10. Chang, Y., McLandsborough, L. and McClements, D. (2013) Physicochemical properties and antimicrobial efficacy of carvacrol nano-emulsions formed by spontaneous emulsification. J. Agric. Food Chem., 61(37): 8906-8913. 
11. Azmi, N., Elgharbawy, A., Motlagh, S., Samsudin, M. and Salleh, H. (2019) Nano-emulsions: Factory for food, pharmaceutical and cosmetics. Processes, 7(9): 617.

12. Kumar, M., Bishnoi, R., Shukla, A. and Jain, C. (2019) Techniques for formulation of nano-emulsion drug delivery system: A review. Prev. Nutr. Food Sci., 24(3): 225-234.

13. Knox, B., Blachowicz, A., Palmer, M., Romsdahl, J., Huttenlocher, A., Wang, C., Keller, N. and Venkateswaran, K. (2016) Characterization of Aspergillus fumigatus isolates from air and surfaces of the international space station. mSphere, 1(5): e00227-16.

14. Abd-Elsalam, K. and Khokhlov, A. (2015) Eugenol oil nano-emulsion: Antifungal activity against Fusarium oxysporum f. sp. vasinfectum and phytotoxicity on cottonseeds. Appl. Nanosci., 5(2): 255-265.

15. Peláez, T., Muñoz, P., Guinea, J., Valerio, M., Giannella, M., Klaassen, C.H. and Bouza, E. (2012) Outbreak of invasive aspergillosis after major heart surgery caused by spores in the air of the intensive care unit. Clin. Infect. Dis., 54(3): e24-e31.

16. Steinmann, J., Buer, J. and Rath, P. (2016) Detection of Aspergillus fumigatus in blood samples from critically ill patients in intensive care units by use of the SeptiFast assay. J. Clin. Microbiol., 54(7): 1918-1921.

17. Kami, M., Murashige, N., Fujihara, T., Sakagami, N. and Tanaka, Y. (2005) The mechanism for low yield of blood culture in invasive aspergillosis; the clinical importance of antigen detection tests revisited. Bone Marrow Transplant., 36(1): 85-86.

18. Garcia-Vidal, C., Viasus, D. and Carratalà, J. (2013) Pathogenesis of invasive fungal infections. Curr. Opin. Infect. Dis., 26(3): 270-276.

19. Chen, J., Yang, Q., Huang, J. and Li, L. (2014) Clinical findings in 19 cases of invasive pulmonary aspergillosis with liver cirrhosis. Multidiscip. Respir. Med., 9(1): 1.
20. Bulpa, P., Dive, A. and Sibille, Y. (2007) Invasive pulmonary aspergillosis in patients with chronic obstructive pulmonary disease. Eur. Respir. J., 30(4): 782-800.

21. Bandres, M., Modi, P. and Sandeep, S. (2019) Aspergillus fumigatus. StatPearls Publishing LLC, Treasure Island, FL.

22. Rehman, F.U., Shah, K.U., Shah, S.U., Khan, I.U., Khan, G.M. and Khan, A. (2017) From nano-emulsions to self-nano-emulsions, with recent advances in self-nanoemulsifying drug delivery systems (SNEDDS). Expert Opin. Drug Deliv., 14(11): 1325-1340.

23. Hussein, J., El-Banna, M., Mahmoud, K., Morsy, S., Latif, Y., Medhat, D., Refaat, E., Farrag, A. and El-Daly, S. (2017) The therapeutic effect of nano-encapsulated and nanoemulsion forms of carvacrol on experimental liver fibrosis. Biomed. Pharmacother., 90: 880-887.

24. Samarghandian, S., Farkhondeh, T., Samini, F. and Borji, A. (2016) Protective effects of carvacrol against oxidative stress induced by chronic stress in rat's brain, liver, and kidney. Biochem. Res. Int., 2016: 2645237.

25. Langeveld, W.T., Veldhuizen, E.J. and Burt, S.A (2014) Synergy between essential oil components and antibiotics: A review. Crit. Rev. Microbiol., 40(1): 76-94.

26. Nguefack, J., Leth, V., Zollo, P. and Mathur, S. (2004) Evaluation of five essential oils from aromatic plants of Cameroon for controlling food spoilage and mycotoxin producing fungi. Int. J. Food Microbiol., 94(3): 329-334.

27. Basak, S. and Guha, P. (2017) Betel leaf (Piper betle L.) essential oil microemulsion: Characterization and antifungal activity on growth, and apparent lag time of Aspergillus flavus in tomato paste. LWT Food Sci. Technol., 75: 616-623.

28. Zhang, J., Ma, S., Du, S., Chen, S. and Sun, H. (2019) Antifungal activity of thymol and carvacrol against postharvest pathogens Botrytis cinerea. J. Food Sci. Technol., 56(5): 2611-2620.

$* * * * * * * *$ 